\title{
Isolated primary cold abscess of the sternum: a case report
}

\author{
Lovenish Bains ${ }^{*}$ (D), Pawan Lal, Tirlok Chand, Kamal Kishore Gautam, Mohd Yasir Beg and Pritesh Kumar
}

\begin{abstract}
Background: Musculoskeletal tuberculosis forms $10-25 \%$ of extrapulmonary tuberculosis which mainly involves the spine or weight-bearing joints. Tuberculous involvement of the sternum is a rare clinical entity even in countries where tuberculosis has high prevalence. Primary tuberculous sternal osteomyelitis accounts for approximately $0.3 \%$ of all types of tubercular osteomyelitis and the probable source appears to be extension from paratracheal or hilar lymph nodes. Despite tuberculosis being a common disease in endemic countries and worldwide, a thorough literature search of the PubMed database for keywords "primary tuberculosis of sternum" and "primary tuberculous osteomyelitis of sternum" yielded 30 and 22 articles, respectively.

Case presentation: We present an unusual case of a large dumb-bell-shaped cold abscess arising due to infection of the sternum. A 23-year-old immunocompetent Asian woman presented with a gradually progressing painless swelling on anterior chest wall for the last 5 months. She had a large visible swelling on anterior chest wall which was $12.5 \mathrm{~cm}$ in diameter, soft, non-tender, temperature was not raised, and fluctuant. Magnetic resonance imaging showed a large dumb-bell-shaped hyperintense collection in upper anterior chest wall with marrow edema and cortical irregularity in left side of manubrium. Pus was positive for nucleic acid testing (cartridge-based nucleic acid amplification test) for Mycobacterium tuberculosis and later culture was also positive. She was started on antitubercular therapy and aspirated twice. Currently, she has completed 6 months of therapy and the swelling has now disappeared.

Discussion: Swelling, pain localized to sternum, or ulceration of the skin with discharging sinus along with or without constitutional symptoms are the usual presentation. A high element of suspicion is needed for early diagnosis and treatment to prevent its complications. Sternal mycobacterial infections are categorized as primary, secondary, and/or acquired postoperatively. Although radiological investigations aid in diagnosis, the diagnosis is established by positive culture or histopathological examination. Anti-tubercular therapy is the mainstay of treatment with standard four-drug regimen for 6-9 months. Surgical drainage of the abscess should be considered only if it does not resolve by aspiration and anti-tubercular therapy.
\end{abstract}

Keywords: Tuberculosis, Sternum, Osteomyelitis, Cold abscess, Anti-tubercular therapy

\section{Introduction}

Skeletal tuberculosis (TB) accounts for $1-4 \%$ of patients with mycobacterial infection [1]. Any bone can be a site for $\mathrm{TB}$, but sternum involvement is very uncommon. Primary sternal TB without pulmonary involvement is even more uncommon. Despite TB being a common disease in endemic countries and worldwide, a thorough literature search of the PubMed database for keywords "primary tuberculosis of sternum" and "primary

\footnotetext{
* Correspondence: lovenishbains@gmail.com

Department of Surgery, Maulana Azad Medical College, New Delhi, India
}

tuberculous osteomyelitis of sternum" yielded 30 and 22 articles, respectively. Primary tuberculous sternal osteomyelitis accounts for approximately $0.3 \%$ of all types of tubercular osteomyelitis and the probable source appears to be extension from paratracheal or hilar lymph nodes [2]. This pattern of disease has been scarcely reported even from the endemic countries with high disease burden [3-5]. We present our experience of a young woman with large dumb-bell-shaped collection originating from the sternum as an isolated primary cold abscess of the sternum. This type of clinical presentation

(C) The Author(s). 2019 Open Access This article is distributed under the terms of the Creative Commons Attribution 4.0 International License (http://creativecommons.org/licenses/by/4.0/), which permits unrestricted use, distribution, and reproduction in any medium, provided you give appropriate credit to the original author(s) and the source, provide a link to the Creative Commons license, and indicate if changes were made. The Creative Commons Public Domain Dedication waiver (http://creativecommons.org/publicdomain/zero/1.0/) applies to the data made available in this article, unless otherwise stated. 
needs a high amount of suspicion for diagnosis and management.

\section{Case presentation}

A 23-year-old woman of Asian descent (Caucasian) presented with a gradually progressing painless swelling on anterior chest wall for the past 5 months, with rapid increase in size in the last 1 month associated with increasing discomfort. She had no significant past history and was immunocompetent. There was no history of contact with Koch's infection or in her family. There was no history of trauma to anterior chest wall or any surgery in the vicinity. She is a housewife and belongs to lower middle socioeconomic class as per modified Kuppuswamy scale. She had no addiction of any kind and received a 1-week course of antibiotics (amoxicillin and clavulanate) before presenting to us. Her Bacillus Calmette-Guérin (BCG) vaccination status was unconfirmed. She was of average build, afebrile, pulse rate 76/ minute, respiratory rate $13 /$ minute, and blood pressure $120 / 84 \mathrm{mmHg}$ at presentation. There was a large visible swelling on anterior chest wall that was $12.5 \mathrm{~cm}$ in diameter, soft, non-tender, temperature was not raised, and fluctuant (Fig. 1). The overlying skin was normal and there was no other significant finding. There was no cervical lymphadenopathy. Chest and abdomen examinations and neurological assessment were essentially normal. Ultrasonography revealed a hypoechoic collection with approximately $180 \mathrm{cc}$ contents. Her laboratory investigations were hemoglobin $9.1 \mathrm{~g} \%$, total leukocyte count of 8600 with $64 \%$ polymorphs and 35\% lymphocytes, and erythrocyte sedimentation rate (ESR) $26 \mathrm{~mm}$. Liver functions and renal functions were within normal range. A chest X-ray was also normal. Magnetic resonance imaging (MRI) showed a hyperintense collection in upper chest wall anterior to sternum measuring approximately $120 \times 68 \times 49 \mathrm{~mm}$ in size (Fig. 2) with marrow edema and cortical irregularity in left side of manubrium (Fig. 3) and another collection in vicinity which was communicating with primary swelling (Fig. 4).

Few enlarged paratracheal lymph nodes were present. The pus was aspirated and subjected to investigations.

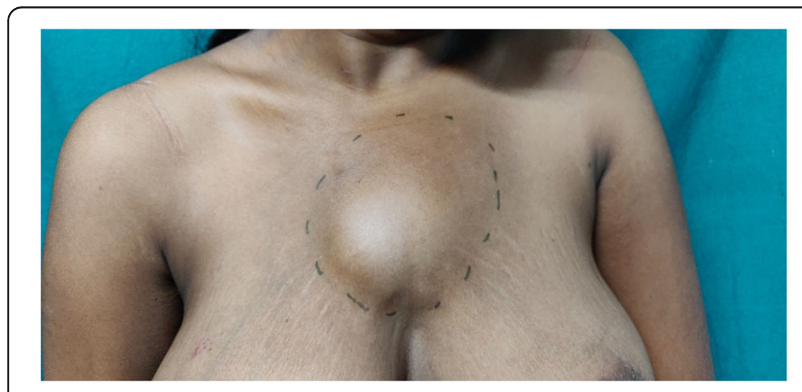

Fig. 1 Large fluctuant anterior chest wall swelling

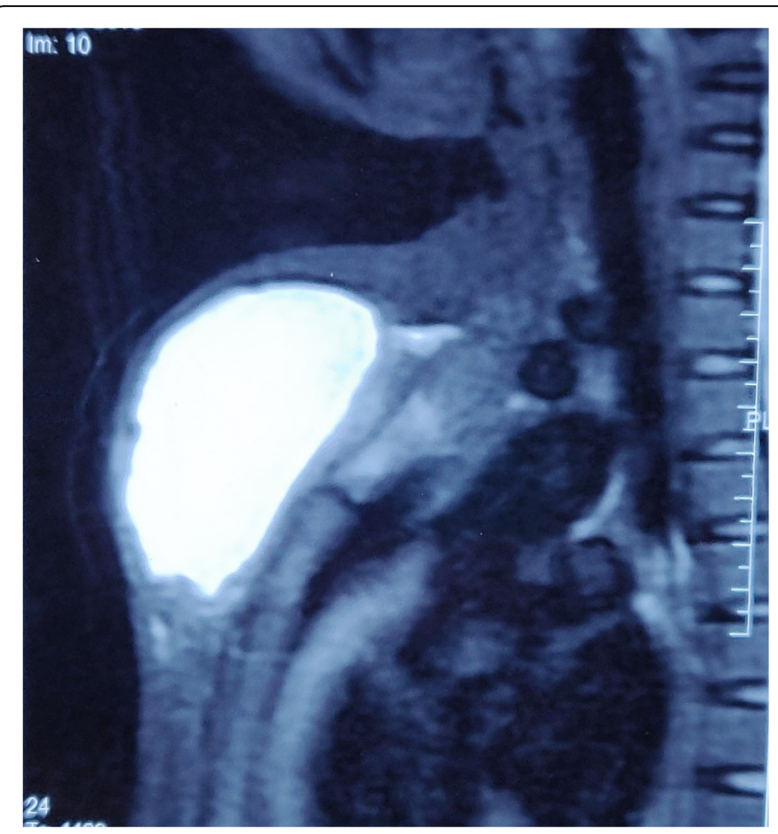

Fig. 2 Magnetic resonance imaging (sagittal plane) showing the collection anterior to sternum

The pus was negative for acid-fast bacilli (AFB) but nucleic acid testing by cartridge-based nucleic acid amplification test (CB-NAAT) for Mycobacterium tuberculosis was positive and susceptible to rifampicin. The pus culture showed no growth after 72 hours. A Mantoux test read at 48 hours was $4 \mathrm{~mm}$; sputum examination and culture were negative for AFB. Her weight was 55 $\mathrm{kg}$ and she was started on daily dose anti-tubercular therapy (ATT) with four drugs, which were isoniazid (300 mg), rifampicin (450 mg), pyrazinamide (1200 mg), and ethambutol $(800 \mathrm{mg})$, for 2 months (2HRZE) followed by 4 months of isoniazid and rifampicin (4HR).

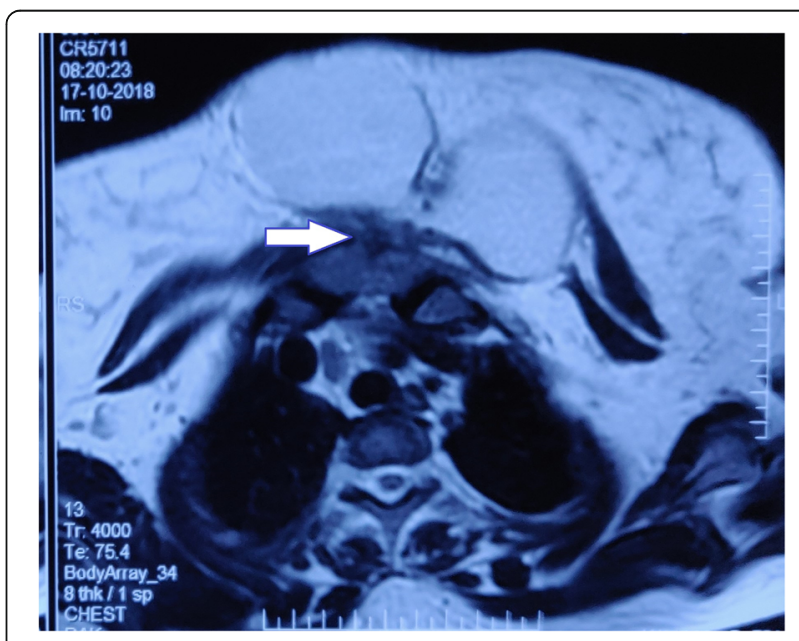

Fig. 3 Cortical breach (arrow) in left side of manubrium 


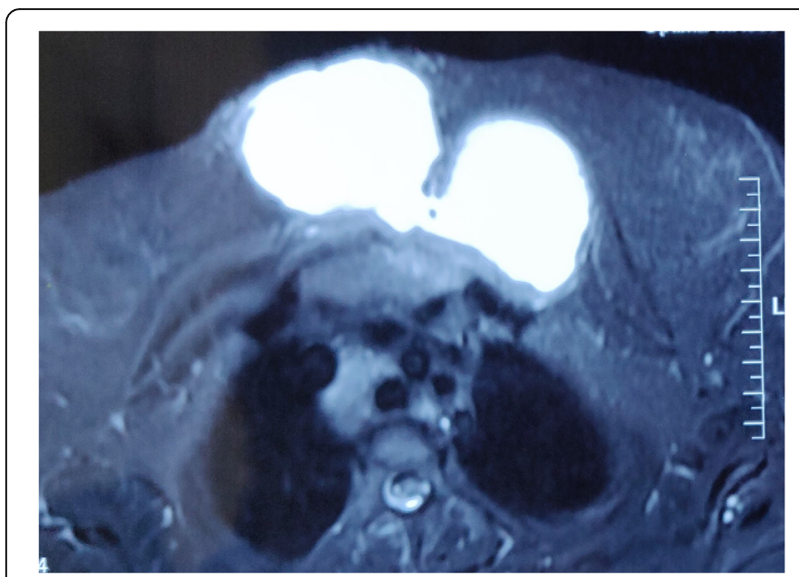

Fig. 4 Magnetic resonance imaging (transverse plane) showing two large communicating collections

She needed two aspirations over 5 weeks initially. The culture became positive for $M$. tuberculosis. After 7 weeks of therapy, the swelling decreased considerably (Fig. 5a). Currently, she has completed 6 months of therapy and the swelling has now disappeared (Fig. 5b).

\section{Discussion}

TB can disseminate to almost every organ after a primary infection or reactivation of latent foci. As per the Global TB report 2018, 10 million new cases occurred in 2017 and ten countries accounted for $80 \%$ of the cases with the top three being India (26\%), Indonesia (11\%), and Nigeria (9\%) [6]. This implies that India accounts for about a quarter of the world's cases of TB [6]. The rate of extrapulmonary TB (EPTB) worldwide has become 10-15\% with young patients, females, and people from Africa or Asia seeming to have a higher risk [5]. Out of all cases of EPTB, 10-25\% have musculoskeletal TB with the most common affected site being the spine (50-69\%), followed by the hip, knee, and ankle/foot (10-13\% each) [7].

Approximately 60 to $80 \%$ of cases of skeletal TB involve the spine or weight-bearing joints, while the sternum is involved in approximately $1 \%$ of cases [7-9]. TB of the sternum is a rare form of flat bone TB that may occur in isolation or in association with pleuropulmonary or lymph node involvement. Isolated primary cold abscess of the sternum becomes a diagnostic puzzle especially if pulmonary TB is not associated. Most of the cases are an extension from a primary focus in the chest and present with ulceration, discharging sinus, or a swelling with constitutional symptoms, whereas a large cold abscess without constitutional symptoms was present in our case.

Sternal mycobacterial infections have been categorized into three types: primary (67.3\%), secondary (20.8\%), and acquired postoperatively (11.9\%) [5]. Tuli and Sinha reported 14 cases of sternal TB in a series of 980 cases of osteoarticular TB (1.5\%) [3]. It mainly involves young males with a mean age of 36 years (range 11 to 59 years), although cases have been found in the pediatric population too [10]. The incidence in males is $65 \%$ whereas in females it is 34\% [5]. Eyer et al. reviewed 27 articles reporting 32 cases from 1966 to 2013 and found that primary sternal TB was more common in men $(76 \%)$ and occurred at a relatively young age [11].

The reactivation of latent loci formed during hematogenous or lymphatic dissemination of primary TB is the primary cause for tubercular sternal osteomyelitis. Direct extension from contiguous mediastinal lymph nodes or infection of retrosternal lymph nodes that erode into the sternum over time are other mechanisms $[5,9,10,12,13]$. Thoracic TB disease most commonly involves the shafts of the ribs or the costovertebral or costochondral junctions whereas lesions of the sternum when found are more common in the manubrium (almost 70\%) than of the body [5]. Yuan found that isolated sternal TB was observed in $60.4 \%$ patients, sternal TB with peristernal tissue invasions (muscles, cartilages, and joints) in $20.1 \%$ patients, and sternal TB with concurrent TB of other organs in 19.5\% patients [5].

The disease often gets detected late due to nonspecific symptoms and slow insidious course. The mean duration of symptoms prior to diagnosis was 6.3 months [11]. The clinical presentation of the disease is variable.

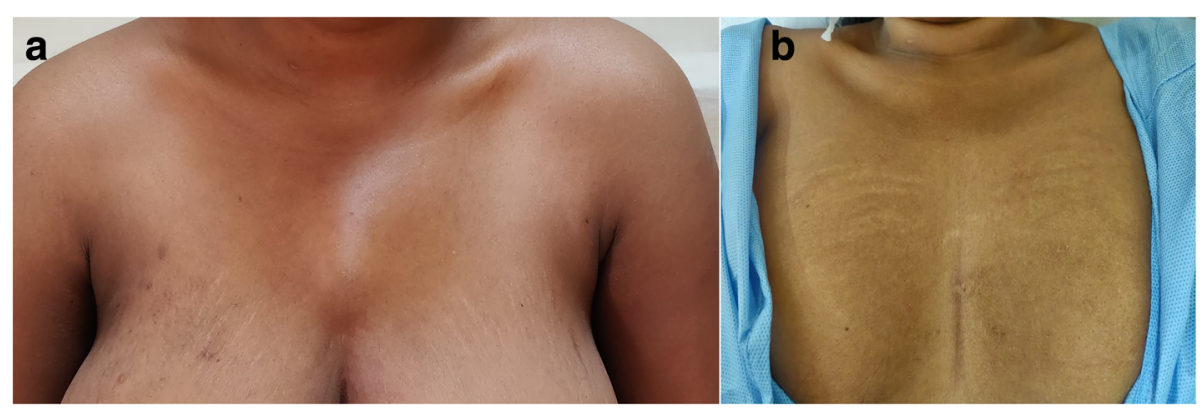

Fig. 5 a Swelling decreased in size after anti-tubercular therapy and aspiration at 7 weeks. b Complete resolution after 6 months of anti-tubercular therapy 
Swelling and pain localized to the sternum are the most common symptoms reported. Others present with ulceration of the skin or discharging sinus. Constitutional symptoms are less commonly seen, but include malaise, fever, night sweats, or weight loss $[2-5,10-12,14]$. In our patient, constitutional symptoms were absent and she had a painless swelling to begin with, which became uncomfortable in the last month due to rapid progression in size. A workup was suggestive of primary sternal TB osteomyelitis and cold abscess.

Blood investigations are essentially normal in most cases apart from raised ESR. Chest radiographs are normal in approximately $70 \%$ of these cases, and approximately $40 \%$ have evidence of $\mathrm{TB}$ in sites other than the sternum, with the lymphatic system being the most common. More than $81 \%$ of cases of sternal TB osteomyelitis have an abnormal tuberculin skin test result $[4,10,11,13,15]$.

According to a study done by Vijay et al., radiological signs may not be present initially at the time of presentation, and symptoms, abscesses, or sinuses may be present long before imaging modalities detect them [16]. Plain radiographs are often normal but radiographic techniques like computed tomography (CT) and MRI are more valuable for localization and detection of bone destruction and soft tissue abnormalities. The common features on $\mathrm{CT}$ of sternoclavicular TB include bone and cartilage destruction, soft tissue masses crossing fascial planes with rim (abscess) and diffuse enhancement (granulation tissue), calcifications, and underlying pleuroparenchymal tubercular involvement [17-19]. MRI delineates abscesses better in the soft tissues, and highlights bone marrow involvement [5, 17-19]. Atasoy et al. demonstrated the role of MRI for early detection of marrow and soft tissue involvement in sternal TB due to the high contrast resolution of MRI [20]. Early changes of cellulitis (seen as replacement of subcutaneous fat signal on T1-weighted images with edema and enhancement) and myositis (showing hyperintensities of the involved muscles on T2-weighted images with their enlargement) are also frequently seen. Late changes are osteomyelitis, joint effusions, and bone destruction. Sinus tract formation, which appears as linear high signal intensity (SI) on T2-weighted images with marginal "tram-track enhancement", may also be seen [17]. Ultrasound is of limited value in early stages but picks up abscesses, osteolytic sternal lesions, or rib lesions later [17].

A needle aspiration or excisional biopsy is mandatory for histopathological diagnosis of sternal osteomyelitis because radiological findings cannot differentiate the cause of osteomyelitis and sometimes may even appear neoplastic [21-23]. The diagnosis is usually confirmed by finding AFB and positive AFB cultures, and caseous necrosis and granuloma on histopathology $[2,3,5,10$,
11, 13, 21]. The frequency of positive cultures is up to $75 \%$. The newer tests like polymerase chain reaction (PCR) amplification and GeneXpert nucleic acid amplification test (NAAT) can also aid the diagnosis in cases of negative smear or culture.

A high index of suspicion is required for early diagnosis and prompt treatment that can prevent complications. ATT is the mainstay of treatment with standard four-drug regimen for 6-9 months. Cold abscess or collections can be aspirated. Khan et al. found that surgical intervention was only necessary if there was: a need for an open biopsy when needle aspiration is inconclusive; draining sinuses; debridement to promote early healing for markedly damaged or sequestrated bones or joint on radiographs, such as pectus excavatum; extensive mediastinal disease or worsening disease; signs of secondary infection or mediastinitis; or disease not responding to an effective course of ATT [19]. The surgical options are needed only for persistent draining sinus and bone destruction which comprises thorough debridement followed by pectoralis major, rectus abdominis, latissimus dorsi, or omental flap closure, with or without chest wall reconstruction or vacuum-assisted closure [19, 24, 25]. The prognosis of the patients is usually good with treatment.

\section{Conclusion}

Isolated primary sternal osteomyelitis due to $M$. tuberculosis is still rare despite the high prevalence of TB in endemic countries. Tubercular involvement of the sternum can occur with various presentations and can involve any age group. It needs a high index of suspicion as diagnosis is usually delayed. A CT scan and MRI provide essential clues but confirmation is by culture or histopathological examination. ATT remains the mainstay of treatment. Surgical drainage of the abscess should be considered only if it does not resolve by aspiration and ATT.

\section{Abbreviations \\ AFB: Acid-fast bacilli; ATT: Anti-tubercular therapy; BCG: Bacillus Calmette- Guérin; CB-NAAT: Cartridge-based nucleic acid amplification test; CT: Computed tomography; EPTB: Extrapulmonary tuberculosis; ESR: Erythrocyte sedimentation rate; M. tuberculosis: Mycobacterium tuberculosis; MRI: Magnetic resonance imaging; NAAT: Nucleic acid amplification test; PCR: Polymerase chain reaction; SI: Signal intensity; TB: Tuberculosis}

\section{Acknowledgements}

Nil.

\section{Authors' contributions}

LB conceptualized the manuscript, reviewed the literature, analyzed data, and made major contribution to the writing of the manuscript. $L B, P L, T C$, $K K G, Y B$, and PK performed the clinical examination, surgical treatment, and clinical follow-up. All authors have read and approved the final version of the manuscript. 


\section{Availability of data and materials}

Not available.

\section{Ethics approval and consent to participate}

Written consent for the publication of this case report was obtained from the patient. Approval for case report by the institutional ethics committee is not required.

\section{Consent for publication}

Written informed consent was obtained from the patient for publication of this case report and any accompanying images. A copy of the written consent is available for review by the Editor-in-Chief of this journal.

\section{Competing interests}

The authors declare that they have no competing interests.

Received: 2 May 2019 Accepted: 30 July 2019

Published online: 25 August 2019

\section{References}

1. Prasad S, Wakhlu A, Misra R, Aggarwal A, Lawrence A, Gupta RK, et al. Features of extra-spinal musculoskeletal tuberculosis: A retrospective study from an North Indian Tertiary Care Institute. Indian J Rheumatol. 2017:12:146-51.

2. Saifudheen K, Anoop TM, Mini PN, Ramachandran M, Jabbar PK, Jayaprakash R. Primary tubercular osteomyelitis of the sternum. Int J Infect Dis. 2010; 14(2):e164-6. https://doi.org/10.1016/j.jijid.2009.03.021. Epub 2009 Jun 12

3. Tuli SM, Sinha GP. Skeletal tuberculosis "Unusual" lesions. Indian J Orth. 1969;3:5-18.

4. Khaira A, Khaira DD, Gupta A, Bhowmik D, Kalra OP, Tiwari SC. Tuberculosis of sternum: three cases with different presentations. J Assoc Physicians India. 2009;57:595-6.

5. Yuan SM. Sternal mycobacterial infections. Ann Thorac Med. 2016;11(2):10311. https://doi.org/10.4103/1817-1737.180032.

6. Global tuberculosis report 2018. https://www.who.int/tb/publications/ global_report/en/ Accessed 5 July 2019

7. Held MFG, Hoppe S, Laubscher M, Mears S, Dix-Peek S, Zar HJ, et al. Epidemiology of Musculoskeletal Tuberculosis in an Area with High Disease Prevalence. Asian Spine J. 2017;11(3):405-11. https://doi.org/10.4184/asj.201 7.11.3.405. Epub 2017 Jun 15

8. Zhao X, Chen S, Deanda A Jr, Kiev J. A rare presentation of tuberculosis. Am Surg. 2006;72:96-7

9. Martini M, Cuahes M. Bone and joint tuberculosis: A review of 652 cases. Orthopedics. 1988:11:861-6.

10. Vasa M, Ohikhuare C, Brickner L. Primary sternal tuberculosis osteomyelitis: A case report and discussion. Can J Infect Dis Med Microbiol. 2009:20(4):e181-4

11. Eyer MM, Constantinescu M, Sendi P. Primary sternal tuberculosis: a case report and review of the literature. JMM Case Reports. 2014;1 https://doi. org/10.1099/jmmcr.0.002063.

12. Sharma S, Juneja M, Garg A. Primary Tubercular Osteomyelitis of the Sternum. Indian J Pediatr. 2005;72:709. https://doi.org/10.1007/BF02724085.

13. Hongsakul K, Chitrapazt N, Tubtawee T, Jaovisidha S. Primary sternal tuberculosis: a case report and literature review. Southeast Asian J Trop Med Public Health. 2015;46(1):80-5.

14. Anand P, Sarin N. Isolated Sternal Tuberculosis Presenting as a Chest Wall Abscess: A Case Report. Iran J Med Sci. 2018;43(4):440-3.

15. Dhillon MS, Gupta RK, Bahadur R, Nagi ON. Tuberculosis of the sternoclavicular joints. Acta Orthop Scand. 2001;72(5):514-7. https://doi. org/10.1080/000164701753532862.

16. Vijay YB, Vinod A, Umesh S, Anubhav G. Primary tuberculous sternal osteomyelitis: a clinical rarity. Asian Cardiovasc Thorac Ann. 2009;17:310-2.

17. Rajput AK, Rajan KE, Gupta RK, Muttagikar MP. Tuberculosis of sternum. Med J Armed Forces India. 2001;57(4):330-2. https://doi.org/10.1016/S0377-123 7(01)80017-4. Epub 2011 Jul 21

18. Shah J, Patkar D, Parikh B, Parmar H, Varma R, Patankar T, Prasad S. Tuberculosis of the sternum and clavicle: imaging findings in 15 patients. Skelet Radiol. 2000;29(8):447-53.

19. Khan SA, Varshney MK, Hasan AS, Kumar A, Trikha V. Tuberculosis of the sternum: A clinical study. J Bone Joint Surg Br. 2007;89:817-20.

20. Atasoy C, Oztekin PS, Ozdemir N, Sak SD, Erden I, Akyar S. CT and MRI in tuberculous sternal osteomyelitis: a case report. Clin Imaging. 2002;26:112-5.
21. Chang GH, Kim SK, Lee WY. Diagnostic issues in tuberculosis of the ribs with a review of 12 surgically proven cases. Respirology. 2009;4:249-53.

22. Rizzo V, Salmasi Y, Hunter M, Sidhu P. Delayed diagnosis of chronic postoperative sternal infection: a rare case of sternal tuberculosis. BMJ Case Rep. 2018;2018 https://doi.org/10.1136/bcr-2017-223650.

23. Calabrò $\mathrm{E}$, Pastorino U. Primary sternal tuberculosis mimicking a lytic bone tumor lesion. Monaldi Arch Chest Dis. 2018;88(1):931. https://doi.org/10.4 081/monaldi.2018.931.

24. Banic A, Ris HB, Erni D, Striffeler H. Free latissimus dorsi flap for chest wall repair after complete resection of infected sternum. Ann Thorac Surg. 1995; 60:1028-32.

25. Ford SJ, Rathinam S, King JE, Vaughan R. Tuberculous osteomyelitis of the sternum: successful management with debridement and vacuum assisted closure. Eur J Cardiothorac Surg. 2005;28:645-7.

\section{Publisher's Note}

Springer Nature remains neutral with regard to jurisdictional claims in published maps and institutional affiliations.
Ready to submit your research? Choose BMC and benefit from:

- fast, convenient online submission

- thorough peer review by experienced researchers in your field

- rapid publication on acceptance

- support for research data, including large and complex data types

- gold Open Access which fosters wider collaboration and increased citations

- maximum visibility for your research: over $100 \mathrm{M}$ website views per year

At BMC, research is always in progress.

Learn more biomedcentral.com/submissions 